\title{
A PESQUISA-AÇÃO EDUCACIONAL NO DESENVOLVIMENTO DOCENTE EM MÉTODOS AVALIATIVOS PARA O MÉDICO RESIDENTE
}

\author{
Bruna de Sá Duarte Auto ${ }^{1}$, Maria Viviane Lisboa de Vasconcelos ${ }^{1}$, Ana Lydia Vasco de \\ Albuquerque Peixoto ${ }^{2}$ e Maria de Lourdes Fonseca Vieira ${ }^{1}$ \\ Faculdade de Medicina da Universidade Federal de Alagoas, Brasil. brunaduarte100@gmail.com; \\ camposdelisboa@gmail.com; vieiramlf@uol.com.br; \\ ${ }^{2}$ Faculdade Seune, Brasil. analydiavet@hotmail.com
}

\begin{abstract}
Resumo. A residência médica é a especialização por excelência na formação médica, e um sistema de avaliação bem elaborado é ferramenta efetiva para garantir a qualificação do futuro especialista. Analisar o sistema de avaliação do médico residente em pediatria de um hospital universitário, com o intuito de promover formação docente em métodos avaliativos. Envolve pesquisa-ação educacional (pesquisa-ensino) realizada com docentes/preceptores da residência médica em pediatria num hospital universitário. As etapas consistiram em: aplicação de questionário semiestruturado sobre os métodos utilizados para avaliar os residentes; realização de workshop sobre avaliação de habilidades clínicas e feedback; aplicação de outro questionário baseado no nível 1 do método Kirkpatrick. A análise dos dados consistiu em estatística simples e análise de conteúdo. Nenhum dos docentes/preceptores utiliza uma avaliação sistematizada de habilidades clínicas, psicomotoras ou afetivas do médico residente. Para tornar o sistema avaliativo mais sólido, é necessária a oferta continuada de treinamento, e, nesse sentido, a pesquisa-ação educacional mostrou-se uma importante contribuição. Instituir a avaliação sistematizada com feedback possibilita identificar e corrigir eventuais desajustes nas habilidades do residente. Adicionalmente, faz-se necessário conscientizar os preceptores do seu papel como formadores/avaliadores.
\end{abstract}

Palavras-chave: Residência Médica; Feedback; Preceptores; Avaliação de Desempenho; Desenvolvimento Docente; Educação Médica.

\section{EDUCATIONAL ACTION RESEARCH IN TEACHER DEVELOPMENT IN ASSESSMENT METHODS FOR RESIDENT PHYSICIANS}

\begin{abstract}
The medical residency is the gold standard specialization in medical training and a wellprepared assessment system is an effective tool to guarantee their qualification. Analyze the assessment system for pediatric residents at a university hospital, in order to promote teacher training in assessment methods. Involves educational action research (teaching-research), carried out with teachers and preceptors of the pediatric medical residency at a university hospital. The steps consisted of applying a semi-structured questionnaire on the assessment methods used with the resident physician; a workshop on clinical skills assessment and feedback; application of another questionnaire based on level 1 of the Kirkpatrick method. Data analysis consisted of simple statistics and content analysis. None of the teachers/preceptors use systematic assessment of the resident physician's clinical, psychomotor or affective skills. In order to make the evaluation system more solid, it is necessary to offer continuous training and, in this sense, educational action research has shown to contribute to this process. Instituting systematic assessment with feedback, makes it possible to identify and to correct any mismatches in the resident physician's skills. It is necessary to make preceptors aware of the importance of their role as a trainer and evaluator.
\end{abstract}

Keywords: Medical Residency; Feedback; Preceptors; Performance Evaluation; Teacher Development; Medical Education. 


\section{INTRODUÇÃO}

A docência na educação médica requer a qualificação do conteúdo da disciplina, a capacidade de preparar o aluno para aprender, interagir com os demais profissionais de saúde e compreender a realidade do Sistema Único de Saúde (SUS), bem como um processo avaliativo bem elaborado, para garantir a qualidade do ensino e o alcance dos objetivos educacionais (Perim et al., 2009).

Diante da complexa atuação do docente/preceptor, a elaboração e a implementação de programas de desenvolvimento docente, atividades que buscam aprimorar os saberes e habilidades dos profissionais de saúde enquanto professores, devem ser consideradas um processo permanente (Perim et al., 2009; Steinert, 2019). Porém, segundo Steinert (2019), apesar do crescente interesse pelo tema nas últimas décadas, poucos trabalhos focaram, especificamente, no desenvolvimento docente para avaliação do aluno.

Um processo avaliativo eficaz requer, além de formação docente para tal tarefa, um sistema que combine diversas formas de avaliação, de modo a atingir todos os elementos do aprendizado (conhecimentos, habilidades e atitudes), além de assegurar a validade e a fidedignidade dos métodos utilizados (Troncon, 2001).

A residência médica é a especialização por excelência na formação do médico, e cabe ao programa assegurar que o egresso atinja o nível almejado de competência (Megale, Gontijo, \& Motta, 2009). Por ser um treinamento em serviço, avaliar, nesse contexto, vai além dos aspectos cognitivos, sendo um desafio diário para o docente e o preceptor.

O cenário desta pesquisa - nossa prática cotidiana enquanto docentes e preceptoras na área de pediatria - é o Hospital Universitário Professor Alberto Antunes (Hupaa), da Universidade Federal de Alagoas (Ufal), que conta com um programa de residência médica (PRM) em pediatria instituído há 30 anos e porta todas as credenciais para mantê-lo vigente até os dias atuais.

No entanto, o processo de avaliação dos residentes do programa ainda apresenta um caráter tradicional e somativo, apesar de a Comissão Nacional de Residência Médica (CNRM) definir diretrizes para a avaliação do residente, desde a resolução CNRM n 05, de 1979 (revogada). Tais diretrizes, reiteradas na resolução CNRM $n^{\circ}$ 02, de 2006, orientam a utilização da avaliação cognitiva e de desempenho, abordando o comportamento ético, o relacionamento 
com a equipe de saúde e com o paciente e o interesse pelas atividades (Brasil, 1979; Brasil, 2006).

O modelo conceitual proposto por Miller há várias décadas, conhecido como pirâmide de Miller, demonstrou aos docentes que, no desenvolvimento profissional, a avaliação não pode ficar restrita aos conhecimentos teóricos, pois é necessário que o estudante saiba aplicar esses conhecimentos, executá-los, de maneira prática, e, finalmente, aplicá-los na vida real (Souza, 2012).

Com base nessas dimensões e no grau de aprendizagem do médico residente, as observações realizadas por docentes e preceptores devem ser direcionadas, além do cognitivo, para as avaliações de desempenho, considerando as habilidades clínicas e psicomotoras, a interação com o paciente, o manejo da informação, a capacidade de julgamento, de síntese e de decisão, bem como a preservação de atitudes éticas (Megale, Gontijo, \& Motta, 2009).

A maioria dos métodos avaliativos de habilidades clínicas tem, como princípio básico, a observação direta do desempenho do residente em tarefas clínicas, em ambiente real ou simulado. Nesse sentido, deve permitir a realização do feedback, preferencialmente imediato (formativo), que consiste em descrever e discutir com o residente a sua performance (Zeferino, \& Passeri, 2007).

Um sistema avaliativo bem elaborado e periódico, com feedback contínuo, é ferramenta efetiva para aprimorar o desempenho do futuro especialista e garantir a sua qualificação, meta de importância indiscutível no processo de formação (Troncon, 2001). Para tanto, a avaliação do residente necessita de sistematização e de institucionalização quanto ao modo de avaliar, além de formação específica para esse importante aspecto do processo de ensinoaprendizagem.

Esta pesquisa propôs responder ao seguinte questionamento: como vêm sendo avaliados os residentes quanto às competências adquiridas no programa de residência médica em pediatria do Hupaa? O objetivo é analisar o sistema de avaliação do médico residente em pediatria de um hospital universitário, com o intuito de promover formação docente em métodos avaliativos. 


\section{PERCURSO METODOLÓGICO}

Para identificar lacunas na prática pedagógica e provocar mudanças nos hábitos educativos, idealizou-se uma pesquisa-ação educacional, devido às suas potencialidades enquanto práxis investigativa, no processo de avaliação do residente, por meio de uma amostra intencional com docentes e preceptores do referido programa de residência em pediatria.

No campo educacional, a pesquisa-ação (pesquisa-ensino), uma abordagem qualitativa de pesquisa em movimento, consiste na investigação sobre a própria prática e implica a tomada de consciência por parte dos participantes, permitindo envolvê-los em todas as fases do percurso metodológico (Zaidan, Ferreira, \& Kawasaki, 2018; Mallmann, 2015; Thiollent, 2002).

Sua utilização no campo educacional permite ao docente pesquisador identificar um problema na sua atividade pedagógica e, através da pesquisa, criar condições para transformá-la, a fim de favorecer o crescimento pessoal e profissional dos pesquisadores e dos participantes envolvidos (Zaidan, Ferreira, \& Kawasaki, 2018; Thiollent, 2002).

A pesquisa-ação segue um ciclo em que se aprimora a prática no movimento entre agir no campo do problema e investigar a respeito dela. O ciclo inclui a identificação do problema e a produção de dados sobre os efeitos de uma mudança na prática, antes e depois da sua implementação, utilizando métodos pré e pós para monitorar os efeitos decorrentes da mudança (Tripp, 2005).

Segundo Malheiros (2011), essa metodologia é muito útil no campo educacional, porque permite estudos quanto a alterações curriculares, metodologias de ensino-aprendizagem, métodos de avaliação, entre outros aspectos.

No desenvolvimento da pesquisa, foram utilizados diferentes procedimentos para a coleta de dados, dividida em três etapas, no intuito de entender a realidade da avaliação do médico residente e de propor estratégias de ação para sua melhoria. Com base no ciclo da pesquisaação, descrevem-se, no fluxograma a seguir, os caminhos percorridos, com as estratégias de investigação e as técnicas de análise dos dados construídas (figura 1). 


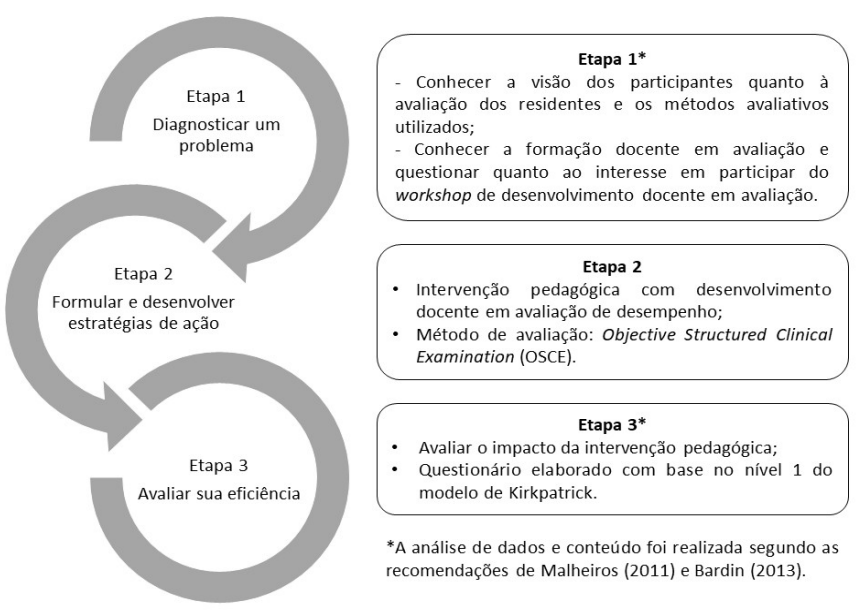

Figura. 1. Fluxograma da pesquisa.

O projeto foi aprovado pelo Comitê de Ética em Pesquisa (CEP) da Universidade Federal de Alagoas, sob o parecer 2.304.092 (CAAE: 74854717.0.0000.5013), não havendo conflitos de interesse.

\subsection{Etapa 1 - Diagnóstico Situacional}

$\mathrm{Na}$ etapa 1 do ciclo da pesquisa-ação, convidaram-se todos os docentes e preceptores que atuavam junto à residência médica em pediatria do Hupaa a participar da pesquisa, perfazendo o total de 22 envolvidos, sendo 16 docentes e 6 preceptores, no período de janeiro de 2018 a abril de 2019. Nessa fase, foi excluído da pesquisa um docente pesquisador, e os demais aceitaram participar na discussão e na resposta ao questionário.

Para identificar as deficiências no processo de avaliação dos residentes, foi aplicado o primeiro questionário semiestruturado, previamente validado e composto de questões referentes aos dados sociodemográficos dos participantes, aos dados específicos sobre capacitação em avaliação, aos métodos avaliativos (testes cognitivos ou avaliação de habilidades clínicas) que utilizam com os médicos residentes, ao conhecimento factual de métodos de avaliação de desempenho em ambientes reais e simulados, à visão dos docentes e preceptores quanto ao processo avaliativo, bem como à intenção de participarem de um workshop de desenvolvimento sobre um método avaliativo. As respostas dos participantes às questões abertas foram organizadas com base nas ideias que emergiram das questões norteadoras, quando se realizou a pré-análise através da leitura mais aprofundada, observando-se a emergência de categorias não criadas previamente. Foi criada uma matriz, e todos os depoimentos foram transcritos ipsis litteris. Os participantes foram codificados por 
letras e números, seguindo a ordem de análise dos questionários - docente (D) e preceptor $(\mathrm{P})$.

Criaram-se matrizes que armazenaram as ideias explícitas ou implícitas, a formação das categorias e as unidades de registro que relacionam os depoimentos com o tema para explicar, no texto, como se chegou ao resultado. Os focos e as unidades de registro foram interpretados, e procedeu-se à elaboração da síntese para cada foco (Malheiros, 2011).

\subsection{Etapa 2 - Intervenção Pedagógica}

Essa etapa consistiu no planejamento de uma intervenção pedagógica, após a análise das respostas ao questionário. Trata-se de uma ação decorrente da busca de uma solução para o problema diagnosticado. Segundo Malheiros (2011), nesse modelo de pesquisa-ação, uma intervenção é realizada para, posteriormente, os seus resultados serem avaliados.

Prosseguiu-se com a construção do workshop de capacitação em avaliação de habilidades clínicas, para os docentes e preceptores, com enfoque no método avaliativo optado pelos participantes. Com o objetivo de manter a estratégia integrativa, as datas para o treinamento foram consensuadas com todos os envolvidos (docentes, preceptores, atores e monitores da pediatria). Todo o processo do workshop foi registrado por meio de fotos e filmagens, com consentimento dos participantes.

\subsection{Etapa 3 - Avaliação da eficácia da intervenção}

A etapa 3 desse ciclo, três semanas após o workshop, caracterizou-se pela avaliação da intervenção pedagógica por meio de um questionário semiestruturado, enviado por correio eletrônico, com base no modelo de Kirkpatrick, que consiste em um modelo de avaliação consagrado internacionalmente e tem a finalidade de avaliar ações educativas voltadas para profissionais. É composto de quatro níveis de avaliação do treinamento: (1) reação, (2) aprendizado, (3) comportamento (transferência) e (4) resultados (J. Kirkpatrick, \& W. K. Kirkpatrick, 2009).

O questionário foi elaborado com base no nível 1 do modelo de Kirkpatrick. Nesse nível, avalia-se a reação dos participantes ao treinamento em si, bem como as suas reações frente à experiência de aprendizado. Foram questionados elementos como o conteúdo programático, as expectativas atingidas, a infraestrutura e a logística (instalações e equipamentos), a duração e a organização do treinamento, a qualidade e o conteúdo do 
material didático, a estrutura dos cenários, assim como a avaliação dos palestrantes (didática, comunicação, interação e conhecimento) e a metodologia utilizada.

\section{RESULTADOS E DISCUSSÃO}

Apresentam-se, a seguir, os resultados quantitativos e qualitativos obtidos nas diferentes etapas do ciclo da pesquisa-ação. Os depoimentos dos docentes e preceptores foram organizados de acordo com os registros de significados, nas categorias "inadequação dos métodos avaliativos", "padronização da avaliação" e "necessidade de feedback".

\subsection{Diagnóstico Situacional}

Os dados coletados no questionário semiestruturado aplicado nessa etapa consideraram o perfil dos participantes e as respostas às perguntas específicas sobre a avaliação dos residentes. Para isso, construiu-se uma amostra intencional com todos os professores e preceptores que atuavam na residência médica em pediatria e que preenchiam os critérios de inclusão, no total de 21 participantes.

Dos participantes, 15 são docentes e 6 são preceptores. $76 \%$ deles (16/21) são do sexo feminino. As idades variam entre 29 e 62 anos (média de 46,5 anos), com tempo de formação entre 7 e 38 anos (média de 22,3 anos). A maior parte do grupo ingressou há mais de 10 anos na universidade, com média de 13,6 anos. Quanto à titulação, todos os participantes são especialistas em pediatria e, além disso, 24\% (5/21) possuem doutorado e 33\% (7/21) possuem mestrado.

Quanto aos métodos avaliativos, 81\% (17/21) dos participantes informaram utilizar mais de um, com finalidade somativa, para obter uma avaliação mais abrangente e fidedigna. No entanto, nenhum dos docentes/preceptores utiliza uma avaliação sistematizada de habilidades clínicas, psicomotoras ou afetivas do residente, nem fornece feedback, utilizando um enfoque mais cognitivo e somativo.

A importância da avaliação de desempenho, nesse nível de formação, deve-se à sua potencialidade para verificar habilidades clínicas (comunicação, exame físico e procedimentos), e a avaliação contínua e formativa permite a correção de falhas e reduz a possibilidade de erros. 
Ross et al. (2018), em um estudo de coorte retrospectivo, analisaram o desempenho e a progressão dos médicos residentes quando avaliados por um sistema tradicional de avaliação somativa, comparado a um sistema de avaliação baseado em competências. Nessa perspectiva, evidenciaram a eficácia da abordagem dos residentes com dificuldades, com foco na possível correção das falhas. Os métodos avaliativos tradicionais identificavam os problemas dos residentes, mas não eram eficazes na correção das lacunas apresentadas, talvez porque as avaliações fossem desconectadas das observações diárias. Nos depoimentos dos docentes e preceptores, surgiram categorias que corroboraram a análise das perguntas objetivas e evidenciaram a preocupação coletiva com a inadequação dos métodos avaliativos: "Ótima pesquisa. Sempre me incomodou a avaliação sem recursos. Acredito que há riscos de injustiça" (D2); "Reconheço a pobreza nos métodos tradicionais de avaliação. Talvez por acomodação e resistência às mudanças ainda não conseguimos sair desta zona de conforto. Aberto a novas experiências" (D6).

Nesta pesquisa, observou-se que o método mais utilizado é a avaliação global, com 76,2\% (16/21) das respostas, seguida da observação do aluno em ambiente real, utilizada por $43 \%$ (9/21) dos participantes. No entanto, todos os que selecionaram essas modalidades de avaliação não as empregam de forma sistemática, com formulários de avaliação (checklists) ou fornecimento de feedback. Quanto à avaliação em ambiente simulado, dois participantes $(9,5 \%)$ relataram alguma formação para esse modelo, embora não o utilizem com os residentes. Os outros instrumentos citados referiam-se a métodos avaliativos cognitivos, como discussão de casos clínicos (10/21 ou 47,6\%), testes de múltipla escolha (7/21 ou 33,3\%) e avaliação oral (7/21 ou 33,3\%).

A avaliação global, o método avaliativo mais utilizado entre os participantes, consiste em uma escala que avalia conhecimento, pontualidade e atitude. Segundo dados da literatura, é o instrumento mais utilizado por todos os cursos de pós-graduação para avaliação de competências nos Estados Unidos (Silber et al., 2004). Contudo, os autores observaram duas grandes limitações da avaliação global. A mais importante delas diz respeito ao fato de que um médico com deficiência em uma área pode atingir uma classificação global satisfatória, se demonstrar destaque em outra competência. A outra limitação é que esse instrumento fornece pouca ou nenhuma informação para um feedback construtivo, um componente importante do desenvolvimento dos médicos residentes (Domingues, Amaral, \& Bicudo-Zeferino, 2009). 
A observação do aluno em ambiente real é um método usual de avaliação, de fácil operacionalização, propício à provisão de feedback imediato e com boa aceitação dos participantes (Troncon, 2001). No entanto, de forma assistemática, sem fornecimento de feedback, gera um baixo grau de fidedignidade.

A avaliação em ambiente simulado permite abordar tarefas clínicas que representam o que é comum na prática médica, em nível de dificuldade compatível com o estágio da formação, e assegurar que todos os alunos sejam avaliados em condições semelhantes, com observação cuidadosa e com a utilização de checklist (Troncon, 2001). No entanto, esse método não é utilizado por nenhum dos participantes junto aos residentes, apesar de dois deles terem conhecimento do mesmo. Esse fato pode sugerir a ausência de um processo institucionalizado de avaliação, a falta de interação entre os participantes e a própria dificuldade para sua operacionalização, por ser um método que demanda o trabalho em equipe, uma execução prolongada e uma logística mais onerosa (cenários, atores, formulários de avaliação).

Utilizar métodos avaliativos de habilidades clínicas, quer em ambientes reais ou simulados, não é uma tarefa fácil, pois envolve a escolha do método mais apropriado e que recaia em uma técnica válida, fidedigna, viável e aceitável por todos os envolvidos no processo (Troncon, 2001). Implica, também, a formação docente em avaliação, o trabalho em equipe, a participação em pesquisas-ação e outras estratégias, além de padronização e sistematização. Alguns participantes manifestaram-se positivamente em relação à pesquisa e à necessidade de padronização da avaliação.

Que a partir dessa pesquisa e dos resultados obtidos, haja uma padronização dos métodos avaliativos utilizados na Famed [Faculdade de Medicina]. É muito importante padronizar as avaliações. (D1)

Ser projeto, cadastrado na gerência de ensino, incluindo preceptores de todas as clínicas. (D4)

É muito importante padronizar as avaliações. (P1)

Na pesquisa, nenhum dos preceptores tinha capacitação formal em avaliação, o que recai sobre o fato de não haver uma exigência de formação pedagógica para o exercício da preceptoria (Botti, 2009), apesar de ser papel do preceptor ensinar, acompanhar na prática diária e avaliar os médicos em formação. Os preceptores, além do bom conhecimento específico na área de atuação, precisam de atividades de desenvolvimento docente e de 
apoio institucional permanente que os auxilie no aprimoramento de suas habilidades de ensino (Almeida, \& Batista, 2011).

A necessidade de treinamento em métodos avaliativos do estudante emerge, nesta pesquisa, quando se questiona se os participantes aceitariam uma capacitação sobre um método avaliativo específico em ambiente simulado e $90 \%$ dos participantes demonstram interesse, como explicitado na fala de P2: "Tenho interesse em capacitações para a docência". Ou, ainda, quando se aborda a necessidade de um método avaliativo de desempenho como o OSCE, por exemplo: "Inserir o OSCE como avaliação integrada no internato de pediatria no $9^{\circ}$ e $10^{\circ}$ períodos (ao menos $1 \mathrm{x} /$ semestre)" (D5).

Um dos depoimentos remete à necessidade de feedback comentada por D3: "Avaliação contínua, no cotidiano, é fundamental para o processo de aprendizagem, sempre com feedback para o seu fortalecimento". Observa-se, nesse depoimento, que o docente sintetiza todo o processo de uma avaliação com função formativa.

O feedback é o substrato de uma avaliação formativa e uma ferramenta efetiva para melhorar o desempenho do estudante, principalmente quando é feito de imediato, após a tarefa clínica (Zeferino, \& Passeri, 2007). Para tanto, deve se dar de forma dialógica, com o aluno desempenhando um papel importante na avaliação do seu próprio desempenho (Ramani, \& Krackov, 2012).

Os resultados mostraram que a preocupação dos participantes quanto aos métodos avaliativos dos residentes era coletiva, considerando-se esse como o primeiro passo para a institucionalização de mudanças dentro do cenário de ensino-aprendizagem. Após a identificação do problema, evidenciado pela ausência de sistematização, institucionalização e formação docente quanto a métodos avaliativos para o residente em pediatria, uma intervenção foi planejada e executada.

\subsection{Workshop: ação formativa, integrativa e interativa}

Com a análise dos resultados dos questionários, um workshop foi elaborado como estratégia de desenvolvimento docente em avaliação. Quando questionados quanto ao método avaliativo que poderia ser explorado, a maioria optou pelo Objective Structured Clinical Examination (OSCE). 
O OSCE é um instrumento de avaliação em ambiente simulado, situado na dimensão "mostrar como faz" da pirâmide de Miller. É um método avaliativo dos mais usados e apreciados em várias partes do mundo. Quando bem elaborado, traz informações importantes para a atuação do futuro profissional.

Após uma revisão da literatura específica sobre métodos de avaliação simulada, elaborou-se um vídeo instrucional sobre o OSCE, que foi utilizado para convidar os docentes e preceptores por meio de um aplicativo de mensagens. Simultaneamente, enviou-se, por correio eletrônico, um material com orientações quanto às atividades que seriam realizadas. No dia da atividade, foi entregue a todos os participantes um manual instrucional sobre o método OSCE, também elaborado pelos pesquisadores.

No workshop com atividade simulada, compareceram 9 (43\%) participantes da pesquisa: 7 docentes e 2 preceptores, pouco menos de $50 \%$ da amostra total, apesar dos horários previamente consensuados. A atividade foi realizada na Faculdade de Medicina da Universidade Federal de Alagoas, localizada ao lado do Hospital Universitário Professor Alberto Antunes. Segundo descrito por Steinert (2019), a proximidade do local de trabalho é um dos fatores facilitadores para aumentar a participação, a motivação e o acesso desses educadores. Dos presentes, 8 eram docentes e preceptores vinculados ao Hupaa. Observouse que apenas um dos seis participantes vinculados às outras instituições compareceu. Quanto aos demais, dois participantes não compareceram por não terem interesse na capacitação, um deles por já conhecer o método, outro pela proximidade da aposentadoria e um último, docente, por estar em pós-doutoramento no exterior. Compareceram, ainda, observadores e convidados, como gestores da residência em pediatria, médicos residentes e monitores de pediatria.

Com o objetivo de possibilitar maior interação e integração com os participantes, o workshop consistiu de dois momentos sequenciais: no primeiro, os pesquisadores expuseram dados gerais sobre avaliação e sobre o método OSCE (em sala de aula); a segunda parte foi direcionada à prática, realizada nas salas de tutoria da Faculdade de Medicina com os atores e manequins do laboratório de habilidades.

No segundo momento, os participantes (docentes, preceptores e observadores) foram direcionados para os cenários do OSCE, portando os formulários de avaliação (checklists) elaborados previamente pelos pesquisadores, com o objetivo de aprimorar suas habilidades em avaliação com a utilização do checklist e com o fornecimento de feedback. Nesse 
momento, nos vários cenários, com os atores (residentes e pessoas convidadas e previamente treinadas) posicionados, iniciou-se o circuito. Os participantes da pesquisa foram orientados a rodiziar pelos vários cenários, em grupos de três ou quatro. Enfatiza-se que o deslocamento nas estações, no OSCE, é feito pelo estudante avaliado, porém, nessa ocasião, o objetivo não foi avaliar o residente, mas possibilitar que o docente e o preceptor participantes pudessem vivenciar uma experiência com diferentes cenários, formulários de avaliação, habilidades clínicas e feedbacks.

Ao final do circuito, os participantes retornaram à sala de aula, onde foram apresentados os passos do OSCE realizado, com os registros fotográficos do workshop.

\subsection{Avaliação da eficácia do workshop}

O nível 1 do método de Kirkpatrick (reação), utilizado na avaliação do treinamento, dimensiona as impressões dos participantes em relação ao conteúdo, aos instrutores, aos materiais e recursos, ao ambiente e às instalações. De acordo com Kirkpatrick (2009), todos os programas devem ser avaliados, nesse nível, como forma de promover melhorias. A reação positiva não garante, necessariamente, a aprendizagem, mas a reação negativa e a insatisfação, com certeza, reduzem as possibilidades de aprendizagem (Mira et al., 2011; Silva, Francisco, Hatakeyama, \& Silva, 2010). Nesse aspecto, os 12 itens da avaliação obtiveram predomínio de avaliações positivas, sendo 10 itens avaliados entre excelente e bom.

No item relativo à aplicação do método avaliativo em treinamento (OSCE), todos os participantes mostraram-se favoráveis a utilizá-lo em sua prática de ensino-aprendizagem com os médicos residentes e, também, com os estudantes da graduação.

$\mathrm{Na}$ atividade, oportunizou-se, também, a participação dos mestrandos da disciplina de avaliação do ensino, como forma de aprendizagem por meio da observação da execução do método em tempo real.

Reforça-se que uma ação positiva, em equipe, sofre uma idealização e gera uma nova ação, conforme os ciclos da pesquisa-ação educacional. Destaca-se, ainda, que o treinamento atingiu o nível 3 de Kirkpatrick, visto que os participantes utilizaram o método avaliativo do aprendizado na sua prática cotidiana, agora junto aos internos. 
O efeito borboleta causado pela intervenção (workshop), na proposição da pesquisa-ação educacional, e pelo método avaliativo em si transformou-se, também, em um ambiente de ensino-aprendizagem para alunos de outros níveis de formação. Espera-se que esse efeito (descrito por Edward Lorenz em 1972), uma metáfora usada na ciência para designar algo causado por uma pequena mudança no início de um evento, possa trazer consequências futuras positivas (Ferrari, Angotti, \& Tragtenberg, 2009) e, ainda, desdobrar-se em novas intervenções em métodos avaliativos na instituição, para todos os níveis de graduação e pósgraduação. E, nesse sentido, é na educação e nas reflexões dela advindas que se produz o efeito borboleta, com potencial para a transformação e para a mudança.

\section{CONCLUSÃO}

Abordar o tema 'avaliação' por meio de uma pesquisa-ação permitiu que os docentes e preceptores pudessem expor suas inquietações quanto ao assunto e reconhecer a fragilidade do processo avaliativo vigente, uma vez que não abrange todos os níveis da pirâmide de Miller, não oferece o feedback e não apresenta sistematização.

A proposta da intervenção, a partir do workshop em um método de avaliação em ambiente simulado, isoladamente, não é suficiente para tornar o processo avaliativo ideal, porém as sensações positivas de participação no planejamento e o empoderamento demonstrado pela prática foram visíveis e favoráveis a intervenções futuras.

A pesquisa-ação, além de implicar a tomada de consciência dos participantes, permite a criação de meios de transformar a prática pedagógica. Nesse sentido, como forma de dar continuidade a um processo de renovação que iniciamos, com o intuito de sistematizar e garantir a fidedignidade do processo avaliativo, sugerimos a construção de uma matriz de competências necessárias ao residente, assegurando que o aluno atinja o comportamento esperado para aquele nível de formação.

Adicionalmente, faz-se necessário conscientizar os preceptores acerca da importância do seu papel como educadores, formadores e avaliadores dos médicos residentes, promovendo maior integração dos eixos ensino-serviço. Dessa forma, o preceptor que acompanha o residente diariamente tem a oportunidade de observar o aluno em atuação, oferecer feedback e identificar e corrigir possíveis erros nas condutas, a fim de contribuir para a qualificação da formação médica. 
Por fim, é necessária a oferta continuada de treinamentos, com o intuito de aprimorar os métodos já utilizados e incluir novos instrumentos avaliativos. A pesquisa-ação educacional mostrou contribuir nesse processo. Embora esses treinamentos signifiquem tempo adicional e custos, eles são vitais para a melhoria da qualidade das avaliações e para o desenvolvimento dos docentes e preceptores nas instituições acadêmicas, além de assegurar a qualidade do pediatra em formação.

\section{REFERÊNCIAS}

Almeida, M. T. C., \& Batista, N. A. (2011). Ser docente em métodos ativos de ensino-aprendizagem na formação do médico. Revista Brasileira de Educação Médica, 35(4), 468-476.

Botti, S. H. O. (2009). O papel do preceptor na formação de médicos residentes: um estudo de residências em especialidades clínicas de um hospital de ensino. (Tese de doutorado), Escola Nacional de Saúde Pública Sergio Arouca.

Brasil. (1979). Resolução CNRM $n^{\circ}$ 05, de 12 de novembro de 1979. Estabelece especialidades médicas credenciáveis como Programa de Residência Médica e dá providências adicionais. Brasília: Ministério da Saúde.

Brasil. (2006). Resolução CNRM nº2, de 17 de maio de 2006. Dispõe sobre os requisitos mínimos dos Programas de Residência Médica e dá outras providências. Brasília: Ministério da Saúde.

Domingues, R. C. L., Amaral, E., \& Bicudo-Zeferino, A. M. (2009). Conceito global: um método de avaliação de competência clínica. Revista Brasileira de Educação Médica, 33(1), 148-151.

Ferrari, P. C., Angotti, J. A. P., \& Tragtenberg, M. H. R. (2009). Educação problematizadora à distância para a inserção de temas contemporâneos na formação docente: uma introdução à Teoria do Caos. Ciênc. educ., 15(1), 85-104.

Kirkpatrick, J., \& Kirkpatrick, W. K. (2009). The Kirkpatrick four levels: a fresh look after 50 years 1959-2009. Kirkpatrick Partners. Recuperado de http://www.kirkpatrickpartners.com/Portals/0/Resources/Kirkpatrick\%20Four\%20Levels\%20white\%20paper. pdf.

Malheiros, B. T. (2011). Metodologia da pesquisa em educação. Rio de Janeiro: LTC.

Mallmann, E. M. (2015). Pesquisa-ação educacional: preocupação temática, análise e interpretação críticoreflexiva. Cadernos de Pesquisa, 45(155), 76-98.

Megale, L., Gontijo, E. D., \& Motta, J. A. C. (2009). Avaliação de competência clínica em estudantes de medicina pelo miniexercício clínico avaliativo (miniex). Revista Brasileira de Educação Médica, 33(2), 166-175.

Mira, V. L., Peduzzi, M., Melleiro, M. M., Tronchin, D. M. R., Prado, M. F. F., Santos, P. T., ... \& Borges-Andrade, J. E. (2011). Análise do processo de avaliação da aprendizagem de ações educativas de profissionais de enfermagem. Revista da Escola de Enfermagem da USP, 45, 1574-1581.

Perim, G. L., Abdalla, I. G., Aguilar-da-Silva, R. H., Lampert, J. B., Stella, R. C. R., \& Costa, N. M. S. C. (2009). Desenvolvimento docente e a formação de médicos. Revista Brasileira de Educação Médica, 33, 70-82.

Ramani, S., \& Krackov, S. K. (2012). Twelve tips for giving feedback effectively in the clinical environment. Medical Teacher, 34, 787-791. 
Ross, S., Binczyk, N. M., Hamza, D. M., Schipper, S., Humphries, P., Nichols, D., \& Donoff, M. G. (2018). Association of a competency-based assessment system with identification of and support for medical residents in difficulty. JAMA Network Open, 1(7), e184581.

Silber, G. C., Nasca, T. J., Paskin, D. L., Eiger, G., Robeson, M., \& Veloski, J. (2004). Do global rating forms enable program directors to assess the ACGME competencies? Academic Medicine, 79(6), 549-556.

Silva, N. P., Francisco, A. C., Hatakeyama, K., \& Silva, M. C. G. (2010). Avaliando as práticas de educação corporativa à distância por meio do modelo de Kirkpatrick: um estudo de caso numa empresa do ramo de energia no estado do Paraná. Emancipação, 10, 501-515.

Steinert, Y. (2019). Faculty development: from rubies to oak. Medical teacher, 41. DOI: 10.1080/0142159X.2019.1688769.

Souza, R. G. S. (2012). Atributos fundamentais dos procedimentos de avaliação. In Tibério, I. F. L. C. et al. (Eds.), Avaliação Prática de Habilidades Clínicas em Medicina, (pp. 2-11). São Paulo: Ed. Atheneu.

Thiollent, M. (2002). Metodologia da pesquisa-ação. São Paulo: Cortez.

Tripp, D. (2005). Pesquisa-ação: uma introdução metodológica. Educação e pesquisa, 31(3), 443-466.

Troncon, L. E. A. (2001). Avaliação de habilidades clínicas: os métodos tradicionais e o modelo "OSCE". Olho mágico, 8(1).

Zaidan, S., Ferreira, M. C. C., \& Kawasaki, T. F. (2018). A pesquisa da própria prática no mestrado profissional. Plurais Revista Multidisciplinar, 3(1), 88-103.

Zeferino, A. M. B., \& Passeri, S. M. R. R. (2007). Avaliação da aprendizagem do estudante. Cadernos ABEM, 3, 3943. 